\title{
PHLÉBOTOMES DU MAYOMBE CONGOLAIS (DIPTERA, PSYCHODIDAE) Étude phénologique.
}

\author{
G. VATTIER-BERNARD* et J. TROUILLET \\ Collaboration technique : Jean-Marie Boudzoumou et André-Simon Bimangou
}

\begin{abstract}
RÉSUMÉ. Le cycle saisonnier d'activité de trois peuplements phlébotomiens du Mayombe congolais se révèle étroitement lié aux facteurs climatiques. L'ensemble du peuplement de la forêt ombrophile "dite de Faustin " présente un cycle d'abondance à deux pics très nettement marqués ; ceux-ci correspondent aux périodes les plus arrosées de l'année. Les espèces de lisière, à Dimonika, manifestent leur maximum d'activité avec la reprise des précipitations. Quant au cycle saisonnier du peuplement de Makaba en 1978, il est nettement perturbé, suite à une sécheresse exceptionnelle en début d'année. Pour chaque espèce, abondance et fréquence sont calculées, les fluctuations saisonnières sont analysées.
\end{abstract}

\section{Phlebotomine sandflies of the Congolese Mayombe (Diptera Psychodidae). Seasonal fluctuations.}

SUMMARY. The seasonal cycle of activities of three phlebotomine sandflies populations of the congolese Mayombe proves to be closely linked to climatic factors. The entire population of the so-called "Faustin" tropical rain forest consist of a cycle of abundance which reaches two very pronounced peaks during the periods of heaviest rainfall each year. The forest-skirt species at Dimonika are most active when the rains begin anew. The seasonal population cycle at Makaba in 1978, on other hand, was severely disrupted following a period of exceptional drought at the beginning of the year. Abundance and frequency are assessed and seasonal fluctuations analysed for each species.

Les Phlébotomes du Mayombe congolais font l'objet de nos recherches depuis plusieurs années. Un premier inventaire a été réalisé (Vattier-Bernard et Trouillet, 1978), des espèces nouvelles ont été décrites (Trouillet, 1982 ; Trouillet et VattierBernard, 1978 ; Vattier-Bernard et Trouillet, 1981 et 1982). Ces résultats acquis, nous avons entrepris l'analyse structurale de trois peuplements phlébotomiens de la région de Dimonika et l'étude phénologique des principales populations ${ }^{1}$. Il nous a paru \footnotetext{
Congo.

* Département de Biologie animale, Faculté des Sciences, B.P. 69, Brazzaville, République Populaire du

I. Travail effectué dans le cadre de l'Équipe Pluridisciplinaire de Biologie forestière de Dimonika.

Accepté le ro février 1983 .
} 
intéressant, en effet, que de telles études déjà réalisées en galerie forestière dégradée et en village de savane dans la région de Brazzaville (Trouillet, 1981) soient effectuées en forêt ombrophile.

\section{Stations de capture et techniques de piégeage (fig. 1)}

Des relevés systématiques ont été faits, selon des techniques de piégeage plusieurs fois utilisées et décrites (Trouillet, 1981 ; Vattier-Bernard et Trouillet, 1978), en trois stations du Mayombe, dans la région de Dimonika :

1) au village de Makaba $\left(4^{\circ} 09^{\prime} \mathrm{S}-12^{\circ} 24^{\prime} \mathrm{E}\right)$ à l'aide d'un piège adhésif lumineux (Rioux et coll., 1969), de mars à décembre 1978, à raison de 8 à 10 captures par mois, de 18 à 6 heures. Ce village est entouré de forêt plus ou moins dégradée ;

2) à Dimonika $\left(4^{\circ} 14^{\prime} \mathrm{S}-12^{\circ} 26^{\prime} \mathrm{E}\right)$, en lisière de forêt ombrophile, avec le même piège, de juin 1980 à octobre 1981, quatre fois par semaine, également de 18 à 6 heures ;

3) en forêt ombrophile, au lieu-dit " forêt Faustin " $\left(4^{\circ} 12^{\prime} \mathrm{S}-12^{\circ} 26^{\prime} \mathrm{E}\right)$ entre Dimonika et Kouilila, de juin 1980 à juillet 1981 (à l'exception du mois de septembre). Dans cette station, nous avons utilisé la capture à main au rythme de deux récoltes de deux heures par semaine. En forêt, le piège adhésif lumineux est peu performant, vraisemblablement à cause de la faible phototaxie des principales espèces forestières.

Au total, 8463 Phlébotomes ont été récoltés.

\section{Données climatiques}

Comme l'ensemble du Congo, la région du Mayombe est soumise à un climat de type équatorial. L'absence ou la présence de précipitations y caractérise deux saisons distinctes : une saison sèche de juin à octobre (températures moyennes mensuelles : $21^{\circ} \mathrm{C}$ ) et une saison des pluies d'octobre à fin mai (températures moyennes mensuelles : 25 à $26^{\circ} \mathrm{C}$ ).

Massif ancien, couvert de forêt ombrophile, le Mayombe est d'altitude modeste (point culminant : $930 \mathrm{~m}$ ) ; toutefois, il constitue face à l'Océan une barrière nettement plus arrosée que les régions voisines. La pluviométrie moyenne annuelle est fréquemment supérieure à $1500 \mathrm{~mm}$ et peut atteindre $2000 \mathrm{~mm}$. Il est rare que les précipitations soient totalement nulles pendant la saison sèche, comme à Brazzaville par exemple.

A noter que nos observations ont été faites, d'une part lors d'une année exceptionnellement sèche (1978) et d'autre part durant une période de pluviosité très moyenne ( $\mathrm{P}=1172,5 \mathrm{~mm}$ d'octobre 1980 à octobre 1981) pour le Mayombe. 


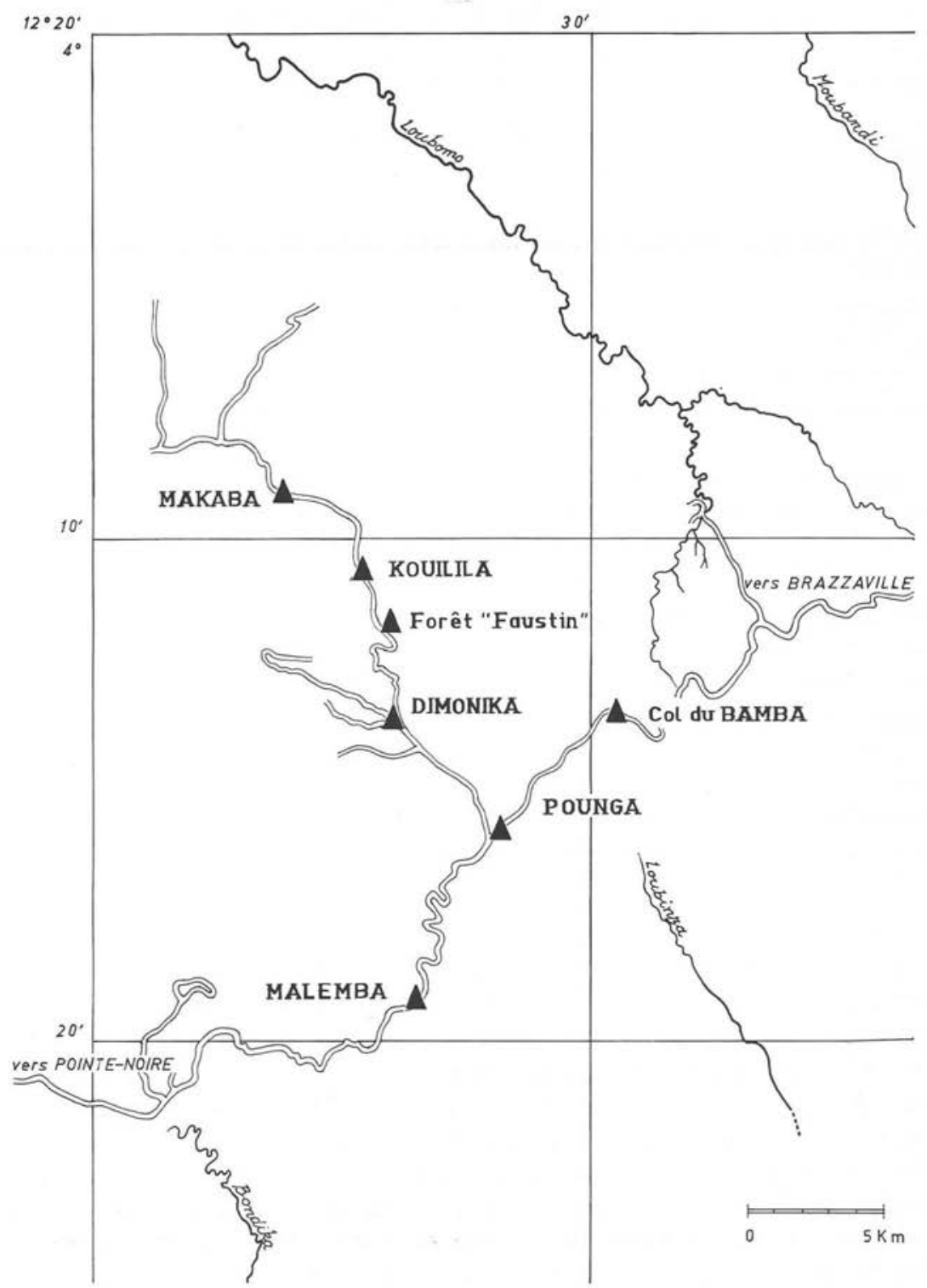

FIG. I. - Stations de capture dans la région de Dimonika. 


\title{
Étude du peuplement de forêt ombrophile
}

\author{
Richesse spécifique, abondance et fréquence (tableau I)
}

La richesse est le nombre d'espèces du peuplement; l'abondance, le nombre relatif des individus de chaque espèce par rapport à l'ensemble des Phlébotomes récoltés ; la fréquence d'une espèce, enfin, s'exprime par le rapport entre le nombre de relevés où celle-ci est présente et le nombre total de relevés. A ce sujet, nous considérons comme :

- très commune : une espèce dont la fréquence est supérieure à $50 \%$;

- commune : une espèce dont la fréquence est comprise entre 25 et $50 \%$;

- rare : une espèce dont la fréquence est comprise entre 10 et $25 \%$;

— très rare : une espèce dont la fréquence est inférieure à $10 \%$.

Tableau I. - Abondance et fréquence des espèces de forêt ombrophile.

\begin{tabular}{lrrc}
\hline \multicolumn{1}{c}{ Espèces } & Abondance (a) & \multicolumn{2}{c}{ Fréquence (f) } \\
\hline S. moreli (Abonnenc et Hamon, 1958) & $44,0 \%$ & $100,0 \%$ & Très commune \\
S. bedfordi* (Newstead, 1914) & $32,4 \%$ & $100,0 \%$ & - \\
S. hamoni (Abonnenc, 1958) & $22,8 \%$ & $74,5 \%$ & - \\
S. ingrami (Newstead, 1912) & $0,7 \%$ & $31,4 \%$ & Commune \\
S. ghesquierei (Parrot, 1929) & $0,08 \%$ & $5,9 \%$ & Très rare \\
S. impudica (Abonnenc, 1968) & $0,05 \%$ & $3,9 \%$ & - \\
S. dissimillima (Abonnenc, 1972) & $0,02 \%$ & $2,0 \%$ & - \\
\hline
\end{tabular}

* Il s'agit de 2 sous espèces confondues : $S$. bedfordi media et $S$. bedfordi ssp.

3655 Phlébotomes ont été récoltés à la main, en un an, dans cette forêt dite de " Faustin ". Sur les sept espèces recensées, trois prédominent nettement : S. moreli, $S$. bedfordi et $S$. hamoni ; elles constituent à elles seules $99,2 \%$ des récoltes et deux d'entre elles sont présentes dans tous les relevés. Au Congo, ces trois espèces sont vraiment inféodées à la forêt ombrophile ; elles sont déjà connues dans la forêt dense de la Haute Sangha (Vattier-Bernard et Bimangou, 1974) dans le massif forestier du Chaillu et dans celui de Bangou (Vattier-Bernard et Trouillet, 1976-1977 a et b). S. moreli est d'ailleurs un excellent indicateur écologique. Moins abondant, S. ingrami est toutefois commun dans ce type de biotope. Quant à la présence de $S$. ghesquierei, $S$. impudica et $S$. dissimillima, elle y est accidentelle.

\section{Fluctuations saisonnières (fig. 2)}

Les Phlébotomes sont présents toute l'année dans la forêt du Mayombe ; il en est de même, du reste, dans la région de Brazzaville. Toutefois, leur activité subit des 

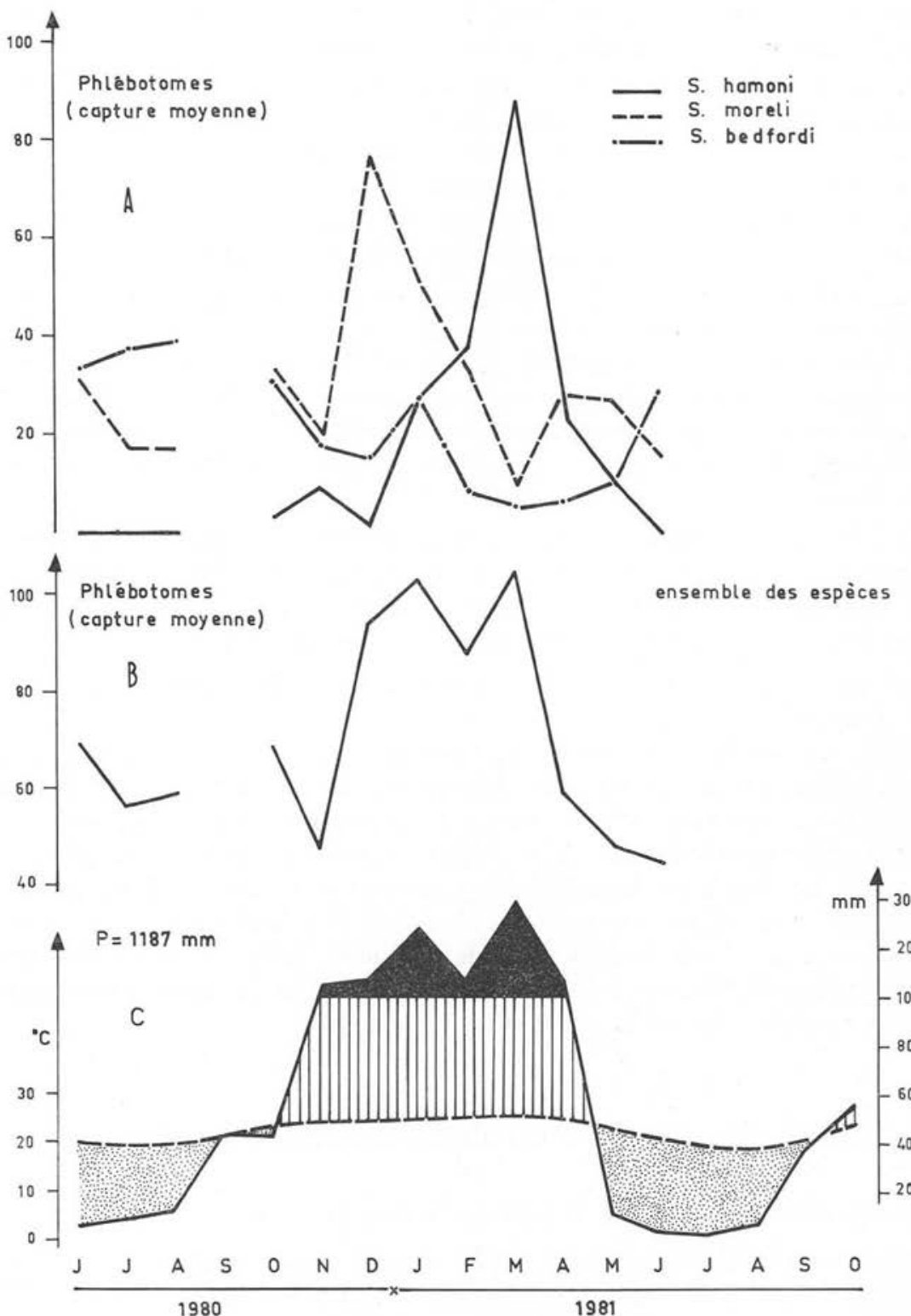

FIG. 2. - A : Fluctuations saisonnières des principales populations de la forêt ombrophile ; B : Fluctuations saisonnières de l'ensemble du peuplement ; C : Diagramme ombrothermique de Dimonika construit suivant la méthode de Bagnouls et Gaussen (1953), modifié par Walter et Lieth (1960, 1967) d'après les données de Clairac, Cros et Ntsila (I980-I98I). Par nuit-piège, il faut entendre les captures effectuées par l'unité-piège ; l'unité-piège correspond à la feuille de papier $20 \mathrm{~cm} \times 20 \mathrm{~cm}$, offrant $800 \mathrm{~cm}^{2}$ de surface efficace. 
fluctuations plus ou moins importantes suivant les espèces et étroitement liées aux facteurs climatiques. La pluviosité notamment semble y jouer un rôle déterminant.

$S$. moreli est relativement abondant toute l'année. Son activité faible au cœur de la saison sèche (juillet-août) s'accroît avec le retour des pluies, passe par un maximum en décembre et atteint son minimum en période de pluviosité maximale (mars). $S$. moreli craint donc autant l'excès que le manque de précipitations. C'est la première fois, à notre connaissance, que la phénologie de cette espèce est étudiée. S. moreli, en effet, est absent des forêts dégradées de la région de Brazzaville.

S. hamoni est très sensible à la sécheresse ; son activité n'est vraiment importante que de janvier à avril ; elle atteint son maximum en mars, et devient nulle en saison sèche. Dans la région de Brazzaville, S. hamoni disparaît totalement des captures avec le début de la saison sèche et ne réapparaît que vers le milieu ou la fin novembre, c'est-à-dire un mois après le retour des pluies. Cette disparition est due à une diapause au stade œuf, que des élevages en laboratoire ont permis de découvrir (Trouillet et Vattier-Bernard, 1979).

$S$. bedfordi est la seule espèce de ce peuplement à bien supporter l'absence de précipitations. Son activité atteint son maximum au cœur de la saison sèche (août) et son minimum lors des pluies intenses de mars. Dans la région de Brazzaville, $S$. bedfordi est l'espèce forestière prédominante en période sèche.

Les quatre autres espèces ont une densité de population trop faible pour faire l'objet d'une telle analyse.

Quant à la courbe illustrant les fluctuations saisonnières de l'ensemble du peuplement phlébotomien de cette forêt ombrophile, elle présente deux pics nettement individualisés et situés au cours des deux mois les plus pluvieux de l'année (janvier et mars) ; le premier pic correspond à l'activité encore dominante bien que décroissante de $S$. moreli, ainsi qu'à une activité déjà importante de $S$. hamoni et non négligeable de $S$. bedfordi; quant au deuxième pic, il traduit l'activité maximale et explosive de $S$. hamoni. Les deux minima, l'un en novembre (début de saison des pluies), l'autre en juin (début de saison sèche), sont essentiellement dus à une activité réduite, très faible ou quasi nulle de $S$. moreli et de $S$. hamoni.

\section{Étude des peuplements de Dimonika et de Makaba}

\section{Richesse spécifique, abondance et fréquence (Tableau II)}

Vingt-deux et dix-huit espèces ont été respectivement récoltées à Dimonika et à Makaba, à l'aide d'un piège adhésif lumineux. Dans les deux stations, distantes d'une dizaine de kilomètres, ce piège était placé en plein écotone. Ainsi, les espèces du village, de la forêt toute proche et de sa lisière pouvaient être attirées.

Dans les deux cas, $S$. ghesquierei domine nettement, suivi de $S$. collarti et de $S$. bedfordi puis de $S$. dureni ou de $S$. decipiens. S. ghesquierei, rarement pris à la main, est un Phlébotome de végétation basse, fréquent en savane; $S$. bedfordi provient de 
la forêt; $S$. collarti et $S$. dureni de la lisière ou du village. La plupart des autres espèces, capturées d'ailleurs en très petit nombre, sont toutes plus ou moins inféodées à la forêt. Notons une fois de plus la très faible phototaxie des espèces sylvicoles : $S$. moreli et S. hamoni.

TABLEAU II. - Abondance et fréquence des espèces à Dimonika et à Makaba.

\begin{tabular}{|c|c|c|c|c|}
\hline \multirow[b]{2}{*}{ Espèces } & \multicolumn{2}{|c|}{ Dimonika } & \multicolumn{2}{|c|}{ Makaba } \\
\hline & $\begin{array}{l}\text { Abondance } \\
(\text { en } \%)\end{array}$ & $\begin{array}{l}\text { Fréquence } \\
(\text { en \%) }\end{array}$ & $\begin{array}{l}\text { Abondance } \\
(\text { en } \%)\end{array}$ & $\begin{array}{l}\text { Fréquence } \\
\text { (en \%) }\end{array}$ \\
\hline S. ghesquierei (Parrot, 1929) & 51,9 & 95,1 & 52,8 & 100,0 \\
\hline S. bedfordi (Newstead, 1914) & 14,8 & 63,8 & 07,7 & 75,0 \\
\hline $\begin{array}{l}\text { S. collarti (Adler, Theodor et Parrot, } \\
\text { 1929) }\end{array}$ & 14,0 & 63,8 & 24,6 & 91,7 \\
\hline S. dureni (Parrot, 1934) & 10,4 & 50,0 & 00,3 & 06,2 \\
\hline S. moreli (Abonnenc et Hamon, 1958) & 2,8 & 28,1 & 1,2 & 29,2 \\
\hline S. ingrami (Newstead, 1914) & 2,1 & 22,8 & 1,7 & 18,0 \\
\hline S. grjebinei (Vattier-Bernard, 1971) & 0,7 & 9,8 & 1,3 & 29,2 \\
\hline S. hamoni (Abonnenc, 1958) & 0,5 & 5,8 & - & 一 \\
\hline S. crosarai (Parrot et Wanson, 1946) & 0,4 & 5,8 & 0,3 & 8,3 \\
\hline S. impudica (Abonnenc, 1967) & 0,3 & 3,6 & 0,7 & 10,4 \\
\hline S. dissimillima (Abonnenc, 1972) & 0,3 & 4,0 & 0,2 & 06,2 \\
\hline S. bernardae (Trouillet, 1982) & 0,2 & 2,7 & 1,3 & 29,2 \\
\hline S. dyemkoumai (Abonnenc, 1964) & 0,2 & 3,1 & 一 & 一 \\
\hline S. emilii (Vattier, 1966) & 0,2 & 3,1 & 0,07 & 2,1 \\
\hline S. decipiens (Theodor, 1931) & 0,2 & 2,7 & 7,1 & 22,9 \\
\hline S. mirabilis (Parrot et Wanson, 1939) & 0,09 & 1,3 & 0,3 & 8,3 \\
\hline $\begin{array}{l}\text { S. moucheti (Vattier-Bernard et Abon- } \\
\text { nenc, 1967) }\end{array}$ & 0,06 & 0,9 & 一 & 一 \\
\hline S. simillima (Newstead, 1914) & 0,06 & 0,9 & 一 & 一 \\
\hline S. serrata (Parrot et Malbrant, 1945) & 0,06 & 0,9 & 0,07 & 2,1 \\
\hline $\begin{array}{l}\text { S. horridula (Vattier-Bernard et Trouil- } \\
\text { let, 1982) }\end{array}$ & 0,06 & 0,4 & 一 & 一 \\
\hline $\begin{array}{l}\text { S. roberti (Vattier-Bernard et Trouillet, } \\
\text { 1981) }\end{array}$ & 0,03 & 0,4 & 0,1 & 4,2 \\
\hline$S$. non déterminé & 0,63 & 一 & 一 & - \\
\hline S. magna (Sinton, 1932) & - & 一 & 0,07 & 2,08 \\
\hline $\begin{array}{l}\text { S. schwetzi (Adler, Theodor et Parrot, } \\
\text { 1929) }\end{array}$ & 一 & - & 0,07 & 2,08 \\
\hline
\end{tabular}


Fluctuations saisonnières ( fig. 3 et 4)

A Dimonika, en 1980-81, l'ensemble du peuplement phlébotomien présente un maximum d'activité très net au mois d'octobre, c'est-à-dire à la reprise des précipitations. L'activité des quatre principales espèces s'effondre après les pluies importantes de janvier, demeure faible en février et en mars. $S$. dureni, $S$. collarti et $S$. bed-

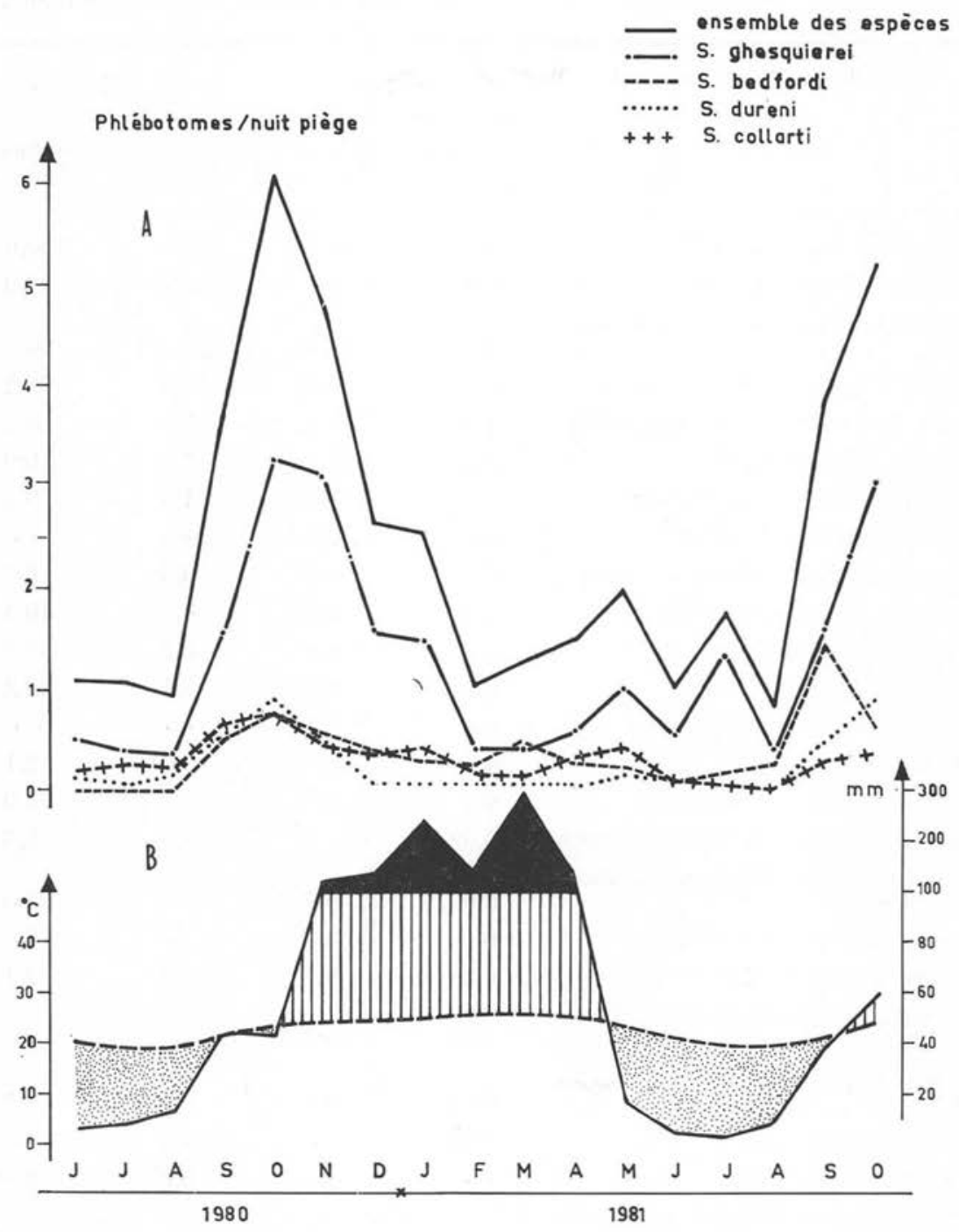

Fig. 3. - A : Fluctuations du peuplement phlébotomien de Dimonika et de ses principales populations; B : Diagramme ombrothermique de Dimonika. 

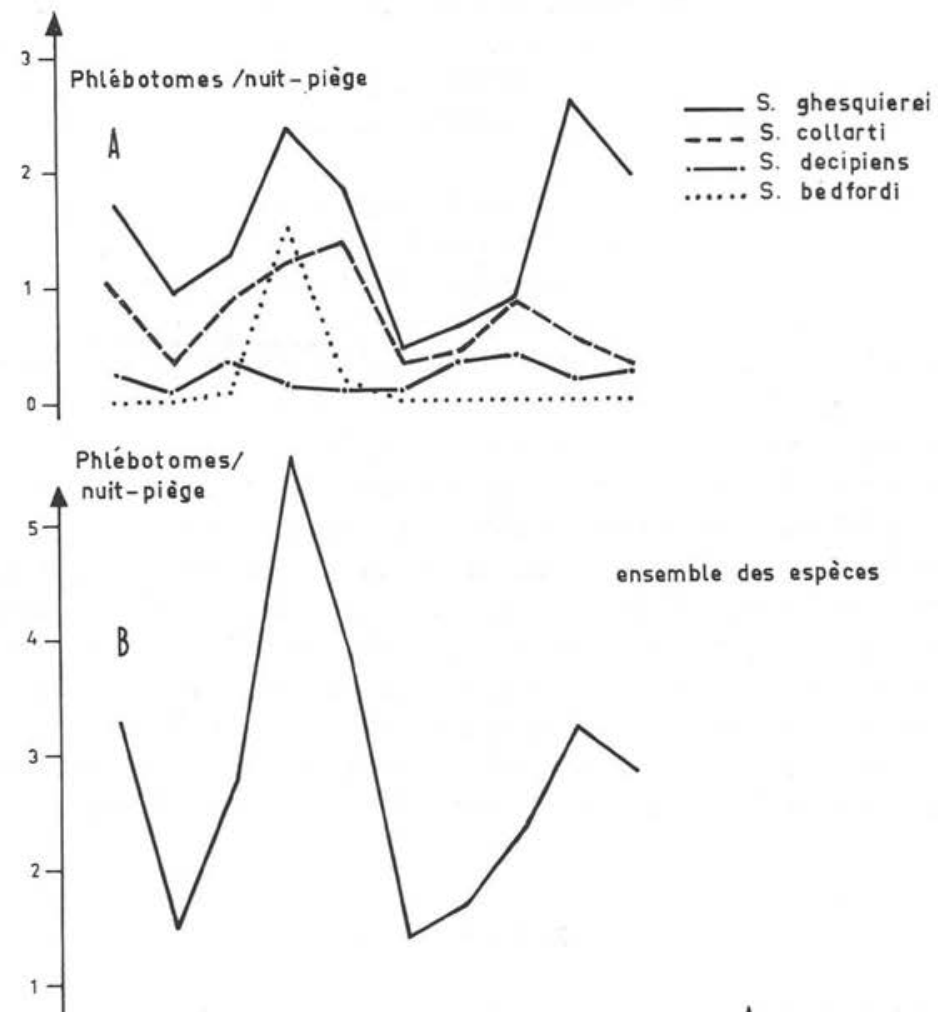

ensemble des espèces

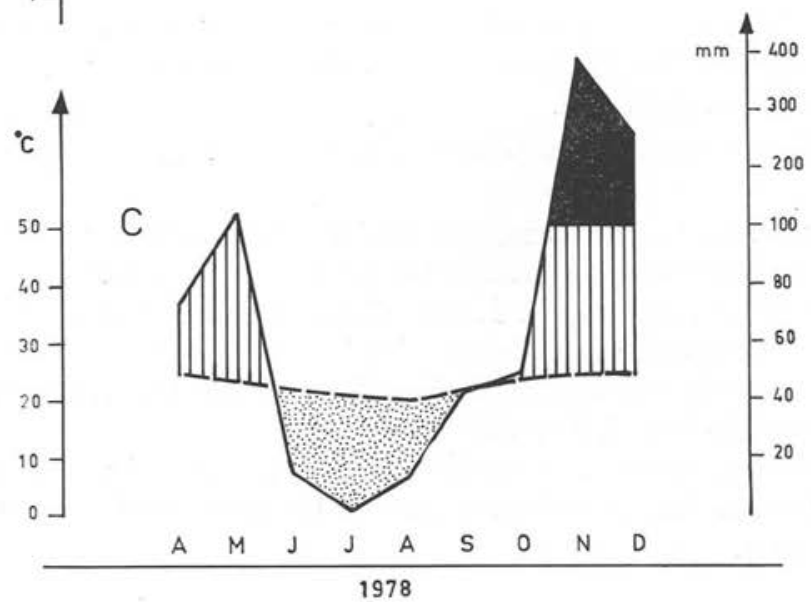

FIG. 4. - A : Fluctuations des quatre principales espèces du peuplement de Makaba ; B : Fluctuations de l'ensemble du peuplement ; C : Diagramme ombrothermique de Dimonika construit d'après les relevés de C. Morin. 
fordi restent peu abondants d'avril à septembre. Quant à l'activité de $S$. ghesquierei, elle est irrégulière et modeste pendant la saison sèche et devient minima au cœur de celle-ci (août). Dans la région de Brazzaville, S. ghesquierei est considéré comme une espèce de saison des pluies disparaissant plus ou moins en saison sèche (Trouillet, 1981).

Il faut également noter que la courbe des fluctuations saisonnières de $S$. bedfordi pris au piège adhésif lumineux n'est pas directement superposable à celle des $S$. bedfordi capturés à la main en forêt ombrophile. A Dimonika, cette espèce présente un maximum d'activité en septembre-octobre ; dans la forêt dite " de Faustin ", celui-ci se situe au mois d'août. Dans les deux cas, toutefois, le minimum est en mars.

Quant aux résultats obtenus deux ans plus tôt au village de Makaba, avec le même système de piégeage, ils peuvent surprendre. Nous tenons à les exposer ici pour montrer combien l'activité des Phlébotomes est liée aux paramètres climatiques et de ce fait peut présenter des fluctuations très variables d'une année à l'autre.

Les premiers mois de 1978 furent exceptionnellement secs ${ }^{2}$ et, comme ce fût le cas dans la région de Brazzaville cette année-là, l'activité des Phlébotomes s'en est trouvée perturbée. Le pic du mois de juin à Makaba en 1978 est lié de toute évidence à la reprise des précipitations $(\mathrm{P}>100 \mathrm{~mm})$ en mai. Quant à celui du mois de novembre, il correspond au début de la nouvelle saison des pluies ; il est légèrement décalé par rapport à celui enregistré à Dimonika en 1980 et 1981 et moins important, conséquence vraisemblable de l'activité exceptionnelle du mois de juin.

\section{Conclusion}

L'ensemble de ces observations révèle nettement que l'activité des Phlébotomes, dans le massif forestier du Mayombe, est étroitement liée aux facteurs climatiques et plus particulièrement à la pluviosité.

Vis-à-vis de ce paramètre, les espèces étudiées présentent des comportements différents :

- S. hamoni est une espèce avide d'eau, très sensible à la sécheresse ; son activité va croissant avec l'importance des précipitations et atteint son maximum en mars ;

- les espèces de lisière, S. ghesquierei, S. dureni, S. collarti ainsi que le Phlébotome sylvicole $S$. moreli, présentent un maximum d'activité durant la première partie de la saison des pluies (octobre et novembre) mais craignent autant les pluies diluviennes (mars) que la saison sèche (juin à septembre) ;

- les preferendum de $S$. bedfordi dans ce domaine sont moins évidents. Cette espèce, toutefois, est la moins sensible à la sécheresse puisqu'elle présente en forêt une activité maxima au mois d'août.

2. Données pluviométriques relevées à M'Vouti $(6,5 \mathrm{~km}$ à vol d'oiseau de Dimonika) $\mathrm{P}=$ $601,9 \mathrm{~mm}$ pour décembre $1976+$ janvier et février $1977 ; \mathrm{P}=71,5 \mathrm{~mm}$ pour décembre $1977+$ janvier et février 1978 . 


\section{BIBLIOGRAPHIE}

Abonnenc E. : Les Phlébotomes de la région éthiopienne. Mém. O.R.S.T.O.M., I972, 5s, 298 p. Bagnouls F., Gaussen H. : Saisons sèches et indices xérothermiques. Doc. Cartes Produc. Végét., $1953,3,8$.

Clairac E., Cros B., Ntsila A. : Caractéristiques climatiques. Dimonika, Congo, I980-I98I.

Rroux J. A., Golvan Y. J., Croset H., Tour S., Houin R., Abonnenc E., Petitdidier M., Vollhardt Y., Dedet J.-P., Albaret J.-L., Lanotte G., Quilici M. : Épidémiologie des Leishmanioses dans le Sud de la France. Monographie de l'I.N.S.E.R.M., 1969, 37, Paris, $221 \mathrm{p}$.

Trouillet J. : Écologie des Phlébotomes du Congo. Thèse Doctorat d'État - Université Paris-Sud, 1981, 381 p.

Trouillet J. : Sergentomyia bernardae n. sp. (Diptera, Psychodidae). Espèce nouvelle récoltée dans le Mayombe congolais. Ann. Parasitol. Hum. Comp., I982, 57, 307-313.

Trouillet J., VATtIer-Bernard G. : Sergentomyia (Sergentomyia) bergerardi n. sp. (Diptera, Phlebotomidae). Espèce nouvelle de la République Populaire du Congo. Ann. Parasitol. Hum. Comp., 1978, 53, 705-709.

Trouillet J., VATTIER-Bernard G. : Cycle annuel de Sergentomyia hamoni Abonnenc, I958 (Diptera, Phlebotomidae) en République Populaire du Congo. Ann. Parasitol. Hum. Comp., I979, s4, 665-672.

VATtiER-BERnARD G., Bimangou A. S. : Contribution à la faune de la République Populaire du Congo. Les collections de l'Université de Brazzavilie. Phlébotomes de la Sangha et de la Cuvette. Ann. Univ. Brazzaville, 1974, Io (C), 91-109.

Vattier-Bernard G., Trouillet J. : Contribution à la faune de la République Populaire du Congo. XII. Phlébotomes (Diptera, Phlebotomidae) de la région du Pool. Inventaire, Répartition géographique et étude écologique. Ann. Univ. Brazzaville, 1976-1977a, $12-13$ (C), $63-84$.

VAtTIER-Bernard G., Trouillet J. : Contribution à la faune de la République Populaire du Congo. XIII. Phlébotomes (Diptera, Phlebotomidae) de la Bouenza, de la Lekoumou et du Niari. Inventaire, Répartition géographique et notes écologiques. Ann. Univ. Brazzaville, 1976-1977 b, $12-13$ (C), 85-ror.

VAtTier-Bernard G., Trouillet J. : Les Phlébotomes (Diptera, Phlebotomidae) du Mayombe (République Populaire du Congo). Ann. Parasitol. Hum. Comp., 1978, 53, 697-704.

Vattier-Bernard G., Trouillet J. : Sergentomyia roberti (Diptera, Psychodidae). Ann. Parasi-

tol. Hum. Comp., I981, 56, 54 I-545.
VAtTIER-Bernard G., Trouillet J. : Sergentomyia horridula (Diptera, Psychodidae), nouveau Phlébotome récolté dans le Mayombe (Congo). Ann. Parasitol. Hum. Comp., I982, 57, 301306.

WALter H., Lieth H. : Klimadiagramweltatlas Fisher Verlag, Iéna, 1960, 1964, 1967, 3 vol. 